\title{
A proibição de chute após a cobrança do lateral ou escanteio modifica o comportamento de jogadores de futsal da categoria sub-09?
}

\author{
Does the prohibition of shooting on goal after the kick-in or corner-kick modify the players' \\ behavior in U-09 futsal?
}

¿La prohibición de tiro directo a la meta después del saque lateral o saque de esquina modifica el comportamiento de los jugadores de futsal de la categoría sub-09?

\author{
Rafael Gomes MacedoI, Pedro Drumond Moreira ${ }^{\mathrm{II}}$, Gibson Moreira Praça ${ }^{\mathrm{III}}$
}

\begin{abstract}
Resumo
O estudo objetivou comparar as propriedades de interação e as finalizações em jogos de futsal da categoria sub-09, com a permissão de gol (CT) e sem a permissão de gol (ST), por meio da "tiradinha" (chute direto ao gol após a cobrança de lateral). Foram analisados 10 jogos formais de futsal, seis ST e quatro CT. A Social Network Analysis e a análise notacional foram utilizadas para as análises. Os resultados apontaram maiores interações entre os jogadores nos jogos CT. Observou-se maior número de finalizações em situações originadas dos laterais e escanteios nos jogos CT. Conclui-se que a presença da "tiradinha", nos jogos de futsal, na categoria sub-09, leva a um aumento da interação entre os jogadores e das finalizações provenientes desta regra. Apesar dos jogos ST apresentarem menores valores nas interações do que CT sugere-se que treinadores possam utilizar esta regra para estimular a progressão dos jogadores na quadra.
\end{abstract}

Palavras-chave: Futsal; Análise de Interações Sociais; Análise Notacional; Finalizações; Ações técnico-táticas

\begin{abstract}
This study aimed to compare the network properties and shots on goal in futsal matches of under-09 teams, in games played with (WiK) and without (WoK) the shooting after the kick-in rule. Six futsal matches in WoK and four in WiK were analyzed. The Social Network Analysis and Notational Analysis were used for interpretation of the network properties and shots on goal, respectively. The results showed higher network values in matches WiK than in matches WoK. The findings of the present study indicated a higher incidence of shots on goal and corner kicks in matches WiK. It is concluded that allowing the players to shot on goal after the kick-in increased the interaction between players and shots on goal in the U-09 futsal matches. Although the WoK showed less network values than $\mathrm{WiK}$, it is suggested that coaches may use this rule to stimulate the progression of players on the court.
\end{abstract}

Keywords: Futsal; Social Network Analysis; Notational Analysis; Shooting on goal; Technical-tactical action

\footnotetext{
${ }^{\text {I } U n i v e r s i d a d e ~ F e d e r a l ~ d e ~ M i n a s ~ G e r a i s ~-~ U F M G ~-~ E n d e r e c ̧ o: ~ A v . ~ P r e s . ~ A n t o ̂ n i o ~ C a r l o s, ~} 6627$ - Pampulha, Belo Horizonte - MG, CEP: $31270-901$ - e-mail: gomes.rafae196@gmail.com

II Universidade Federal de Minas Gerais - UFMG - e-mail: pedrodrumondmoreira@hotmail.com

${ }^{\text {III } U n i v e r s i d a d e ~ F e d e r a l ~ d e ~ M i n a s ~ G e r a i s ~-~ U F M G ~-~ e-m a i l: ~ g i b s o n ~ m o r e i r a @ y a h o o . c o m . b r ~}$
} 


\section{Resumen}

El estudio tuvo como objetivo comparar las propiedades de interacción y las finalizaciones en partidos de futsal sub-09 con la concesión del gol (CT) y sin la concesión del gol (ST) a través de la tiradinha (tiro directo a la meta después del saque lateral). Se analizaron diez juegos formales de futsal, seis ST y cuatro CT. Se adoptaron el Social Network Analysis y el análisis de notación. Los resultados mostraron un valor de interacción más alto en los juegos de CT. Hubo un mayor número de finalizaciones en situaciones que se originaron en laterales y tiro de esquinas en los juegos CT. Se concluye que la tiradinha en futsal en la categoría sub-09, conlleva un aumento de la interacción entre jugadores y de las finalizaciones derivadas de esta regla. Aunque los juegos ST tienen valores más bajos de interacciones que CT se sugiere que los entrenadores pueden usar esta regla para estimular la progresión de los jugadores en la cancha.

Palabras clave: Futsal; Análisis de interacción social; Análisis de notación; Finalizaciones; Acciones técnicotácticas

\section{Introdução}

Praticantes iniciantes no futsal realizam, frequentemente, uma manobra ensaiada caracterizada pela finalização ao gol, a partir do passe recebido após os laterais e os escanteios ofensivos, ação conhecida como "tiradinha" (FARIAS, 2016). A principal vantagem desta manobra é a facilidade do jogador conseguir atingir a meta adversária, com menor pressão dos adversários, devido à exigência de uma distância mínima de 5 metros dos adversários nas bolas paradas (CBFS, 2015). Diante disso, treinadores comumente selecionam os jogadores com melhor habilidade na ação de chute para a realização da "tiradinha", o que pode se revelar uma estratégia eficaz para se chegar ao gol nas categorias iniciais do futsal em razão da baixa estatura dos goleiros (FARIAS, 2016). Isso, porém, poderá impactar no processo de formação dos jogadores, já que essa estratégia conduzirá para as oportunidades de ações em apenas um único jogador da equipe, para o direcionamento do aperfeiçoamento precoce do chute (especialização precoce) no processo de treino, bem como para jogadas ensaiadas rígidas, restringindo a liberdade de ações dos jogadores. Além disso, enfatizar a busca pelo gol por meio da "tiradinha" pode reduzir a diversidade de estímulos aos quais os praticantes são expostos durante o jogo, o que tem sido considerado fundamental no desenvolvimento da criatividade dos jogadores (CASO; VAN DER KAMP, 2020).

Estudos amparam-se na análise notacional para compreender o comportamento de jogadores e equipes no futsal (CASTAGNA e colaboradores, 2009; DOGRAMACI; WATSFORD; MURPHY, 2011; LAPRESA e colaboradores, 2013). Nesta análise, destaca-se o padrão de obtenção de finalizações como um dos principais indicadores na análise do jogo no futsal (AGRAS; FERRAGUT; ABRALDES, 2016). Especificamente, observa-se, na categoria sub-13, maior incidência de finalizações nos setores laterais da quadra, com menor eficácia em relação às categorias sub-15 e sub-17, as quais, por sua vez, apresentaram 
maior incidência de chutes nas áreas próximas ao gol (CABRAL e colaboradores, 2013; PRAÇA; CABRAL; GRECO, 2013). A elevada diferença maturacional entre os jogadores das categorias mais novas (por exemplo, sub-11 e sub-12) (MALINA e colaboradores, 2000) gera confrontos desbalanceados entre bons finalizadores (usualmente atletas mais maturados) e baixos goleiros, facilitando a obtenção do gol, mesmo a partir de finalizações em áreas desfavoráveis da quadra (laterais). Por outro lado, quando o nível maturacional é equilibrado (por exemplo, sub-17), a vantagem da finalização a partir da "tiradinha" é reduzida, o que leva os jogadores a buscarem finalizações em setores mais favoráveis como o centro da quadra (CABRAL e colaboradores, 2013; PRAÇA; CABRAL; GRECO, 2013) .

Além da análise notacional, a Social Network Analysis (SNA) representa uma importante ferramenta de análise das características das interações da equipe (CLEMENTE; MARTINS; MENDES, 2016). As interações são frequentemente medidas por meio da realização de passes (CLEMENTE; MARTINS; MENDES, 2016), o que permite compreender como as equipes gerenciam a posse de bola durante jogos e treinos. Por exemplo, em equipes de jovens praticantes (sub-12, sub-14 e sub-16), observa-se maior proeminência de defensores e alas (MACHADO e colaboradores, 2016). Além disso, na escala coletiva, equipes trocam mais passes quando enfrentam adversários que posicionam as linhas defensivas próximas a própria meta (TRAVASSOS e colaboradores, 2016).

Diante do impacto da manobra ensaiada "tiradinha" na dinâmica do jogo, sugere-se que esta ação impacte o desenvolvimento de aspectos técnicos-táticos de jovens jogadores de futsal. Até o presente momento, nenhum estudo investigou o impacto dessa ação no futsal. Desta forma, utilizar a análise notacional no presente estudo permitirá ampliar o entendimento do impacto da "tiradinha" nas finalizações em diferentes indicadores. Especificamente, considerando que a "tiradinha" permite alcançar o gol adversário com menor número de passes, sugere-se que os jogos em que esta ação é permitida apresentem menores valores de interação (troca de passes) do que os jogos em que esta ação é proibida pelo regulamento da competição. A combinação das informações obtidas por meio do SNA e da análise notacional nas categorias inicias do futsal poderá contribuir em uma melhor estruturação de jogos e treinos, beneficiando a formação esportiva.

Assim, o objetivo do presente estudo foi comparar as propriedades de interação macro, por meio da SNA, e as ações de finalizações em diferentes indicadores: setores da quadra, situação de jogo e resultados das ações de chute, em jogos de futsal da categoria sub-09 com e sem a permissão da regra da “tiradinha". Hipotetiza-se que os atletas executem mais passes, apresentando maior nível de interação nos jogos sem a permissão da "tiradinha". Além disso, espera-se que, nos jogos com a permissão, os jogadores executem mais finalizações nos setores laterais da quadra, com menor eficácia em relação aos 
jogos sem a permissão. Ainda, espera-se que os jogadores possam finalizar nas áreas próximas à meta adversária, nos jogos sem a permissão da "tiradinha".

\section{Materiais e Métodos}

\section{Amostra}

Foram analisados 10 jogos formais de futsal da categoria sub-9 masculina, sendo seis jogos válidos pelo Campeonato Metropolitano Escolar de futsal, competição que prevê, em seu regulamento, a proibição da ação da "tiradinha", e quatro jogos válidos pela Liga Belo Horizonte de futsal, nos quais o uso da "tiradinha" não era limitado pelo regulamento (CT).

Nos jogos em que não havia a permissão da "tiradinha", os gols foram cancelados, quando se executou o chute sem a ação da recepção (domínio) proveniente dos laterais ou escanteios. Nos dois formatos de jogos, todos os jogadores e treinadores possuíam o conhecimento acerca de todas as regras antes dos jogos. Todos os jogos foram de campeonatos de nível regional. Participaram 11 equipes da cidade de Belo Horizonte, sendo seis clubes vinculados à Federação Mineira de Futsal e cinco à Federação Escolar. As diferenças acerca do número de jogos entre os grupos (ST e CT) devem-se ao formato classificatório específico de cada competição. No grupo CT, três equipes foram analisadas em dois jogos e uma equipe foi analisada em quatro jogos. No grupo ST, uma equipe foi analisada em dois jogos e uma equipe foi analisada em três jogos. Contabilizaram-se 120 minutos de jogo analisado para cada grupo. Devido às especificidades da competição, todos os jogos do grupo ST tiveram duração de 30 minutos. Com isso, foram excluídos da análise os 10 minutos finais dos jogos do grupo CT. O projeto tem parecer favorável do Comitê de Ética em Pesquisa (19596019.9.0000.5149).

\section{Procedimentos}

Inicialmente, realizou-se a filmagem dos jogos, por meio do aparelho celular Samsung J7 Pro, modelo SM-J730G, com resolução de vídeo Full HD. Quatro jogos ocorreram no período da manhã e o restante no período da noite. Os jogos ocorreram entre maio e setembro de 2019. Após as filmagens, os jogos foram transferidos para o notebook Dell (Inspiron i3) para análise das ações de passes e chutes. 


\section{Instrumentos}

\section{Social Network Analysis}

Para as análises das interações macro (equipe) dos jogos consideraram-se as variáveis: densidade, que representa a relação entre o número de interações estabelecidas e o número total de interações possíveis, variando entre 0 (mínimo) e 1 (máximo)(CLEMENTE; MARTINS; MENDES, 2016); e dispersão do Eigenvector, que indica se as interações em uma equipe são mais heterogêneas ou homogêneas, variando entre 0 (mais homogênea) e 1 (mais heterogênea) (CLEMENTE; MARTINS; MENDES, 2016). Nessas análises, contabilizaram-se os passes bem sucedidos, definidos como os passes que chegaram ao companheiro de equipe sem sofrer interferência dos adversários. Desta forma, cada passe executado representou uma interação entre os jogadores. Nos casos de substituição, o novo jogador substituiu o antigo na planilha, mantendo-se sempre o mesmo número total de avaliados por instante de jogo. No caso de substituição de todos os jogadores durante o intervalo das partidas, os jogadores foram substituídos para análise de acordo com seu posicionamento em quadra. Os goleiros foram incluídos na análise. Ao final, formaram-se as matrizes de adjacência de cada time, como a apresentada abaixo (Figura $1)$.

Figura 1: Matriz de adjacência

\begin{tabular}{|c|c|c|c|c|c|}
\hline & 1 & 2 & 3 & 4 & 5 \\
\hline 1 & 0 & 0 & 0 & 0 & 0 \\
\hline 2 & 3 & 0 & 8 & 3 & 8 \\
\hline 3 & 7 & 21 & 0 & 19 & 3 \\
\hline 4 & 4 & 13 & 7 & 0 & 6 \\
\hline 5 & 0 & 7 & 14 & 15 & 0 \\
\hline
\end{tabular}

Fonte: Autores

Após feita a tabela, os dados centrais foram copiados e salvos em documentos no formato .txt, que foram exportados para o programa Social Network Analysis SocNetV, versão 1.9, para extração dos dados. 


\section{Análise notacional}

Por meio da análise notacional, contabilizou-se a frequência dos chutes ao gol, que foram considerados como ações com clara intenção de se fazer o gol. Baseado na proposta de Praça, Cabral e Greco (2013), a partir dos critérios estabelecidos por Silva e colaboradores (2013), a análise das ações de chute foi feita por meio de três indicadores de jogo: situação - jogo organizado, contra-ataque, lateral, escanteio, falta e bola roubada; setor - 1 a 10, sendo os setores 1, 4 e 7 laterais pela esquerda, 2, 5 e 8 centrais, 3, 6 e 9 laterais pela direita e o 10 a meia quadra defensiva (Figura 2); e resultado das ações de chutes - gol, trave, defendida pelo goleiro, rebatida pelo goleiro, interceptada pela defesa e fora.

Figura 2 - Setores de Finalização

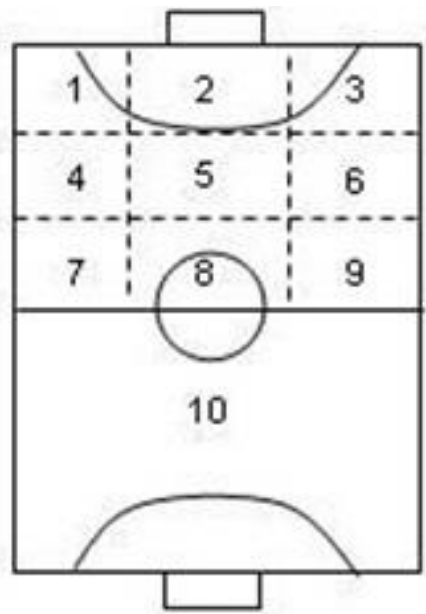

Fonte: Praça, Cabral e Greco (2013)

\section{Qualidade dos Dados}

Para a confiabilidade dos dados, reanalisaram-se dois jogos (25\%) (TABACHNICK; FIDELL; ULLMAN, 2007), adotando os procedimentos intra e inter avaliadores. As reanálises ocorreram após 21 dias, de forma a minimizar a familiaridade dos observadores com as cenas avaliadas. A análise da confiabilidade ocorreu por meio do cálculo do Coeficiente de Correlação Intraclasse (CCI). A análise revelou valores excelentes de confiabilidade CCI intra avaliador $=0,99(0,1)$ e CCI inter avaliador $=0,98$ $(0,1)$ (LANDIS; KOCH, 1977). 


\section{Análise de Dados}

Os dados foram reportados por meio da média e do desvio-padrão das variáveis. Utilizou-se o teste Shapiro-Wilk para verificar a normalidade dos dados. Para a análise das variáveis de interação (SNA), recorreu-se ao teste-t independente (ST x CT). Analisou-se o tamanho de efeito, utilizando o teste $\mathrm{d}$ de Cohen, classificado em pequeno $(0,20)$, moderado $(0,50)$ ou grande $(0,80)$ (COHEN, 1988). Para a análise das ações de chute, realizaram-se anovas two-away de desing misto (ST/CT x setores das finalizações; ST/CT x origem das finalizações; ST/CT x resultado das finalizações). Para as variáveis que não atenderam aos pressupostos de esfericidade, aplicou-se a correção de Greenhouse-Geisser. Verificadas diferenças, recorreu-se ao teste post-hoc de Bonferroni. Para esse procedimento, adotou-se o nível de significância de 5\%. Além disso, calculou-se o tamanho do efeito Eta quadrático parcial $\left(\eta^{2} p\right)$ conforme recomendações da literatura, classificado em "sem efeito" $\left(\eta^{2} p<0,04\right)$, "efeito mínimo" $(0,04$ $\left.<\eta^{2} \mathrm{p}<0,25\right)$, "efeito moderado" $\left(0,25<\eta^{2} \mathrm{p}<0,64\right)$ e "efeito forte" $\left(\eta^{2} \mathrm{p}>0,64\right)$ (FERGUSON, 2009). Para todas as análises, recorreu-se ao software SPSS (Statistical Package for Social Science) for Windows®, versão 19.0.

\section{Resultados}

\section{Interações}

Conforme a tabela 1 , apenas os valores de densidade apresentaram diferença significativa $(\mathrm{p}=0,03$; efeito pequeno) com maior média reportada nos jogos CT.

Tabela 1: Médias (desvio-padrão) de Densidade e Eigenvector nos dois grupos (ST e CT).

\begin{tabular}{lcc}
\hline Campeonato & Densidade & Eigenvector \\
\hline ST & $0,7625(0,025)$ & $0,419(0,122)$ \\
\hline CT & $0,8125(0,025)$ & $0,431(0,12)$ \\
\hline p-valor & $0,030^{*}$ & 0,760 \\
\hline Tamanho do efeito & 0,30 & 0,14 \\
\hline
\end{tabular}

Legenda: * = Diferença Significativa; ST = sem a permissão da "tiradinha"; CT= com a permissão da "tiradinha". 


\section{Análise notacional: setores, origem e resultado das finalizações}

A tabela 2 apresenta a média (desvio-padrão) das finalizações entre os setores e a condição do jogo (ST e CT). Inicialmente, não se observou efeito de interação entre as variáveis $\left(\mathrm{p}=0,08 ; \eta^{2} \mathrm{p}=0,16\right.$; efeito pequeno). Entretanto, observaram-se valores significativos para o efeito principal da condição do jogo $\left(\mathrm{p}=0,001 ; \eta^{2} \mathrm{p}=0,27\right.$; efeito moderado), com maior média para os jogos CT. Verificaram-se também valores significativos para o efeito principal setores $\left(\mathrm{p}=0,001 ; \eta^{2} \mathrm{p}=0,68\right.$; efeito forte), com maior média de finalizações nos setores centrais (2 e 5) e nos setores laterais (3,6,7 e 9).

Tabela 2: Médias (desvio-padrão) das finalizações dentro dos setores.

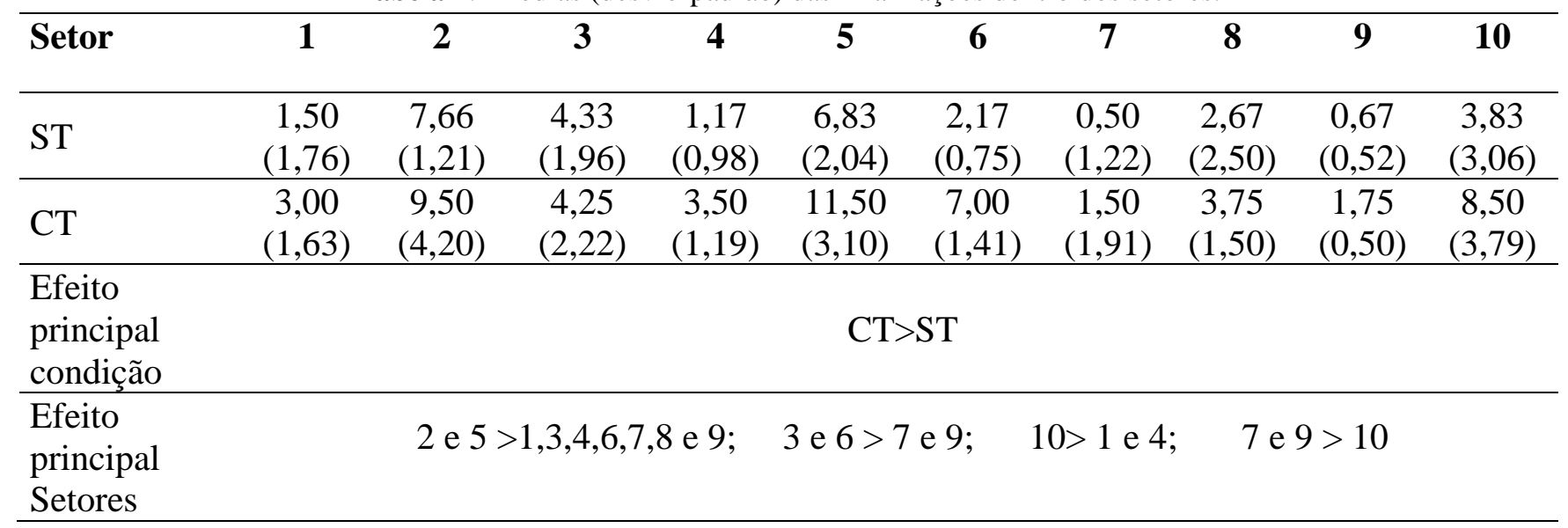

Legenda: ST = sem a permissão da "tiradinha"; CT= com a permissão da "tiradinha".

Fonte: Autores

Conforme a tabela 3 , não se observou efeito de interação entre as variáveis $\left(p=0,2 ; \eta^{2} p=0,13\right.$; efeito pequeno). Entretanto, observaram-se valores significativos para o efeito principal da condição do jogo $\left(\mathrm{p}=0,001 ; \eta^{2} \mathrm{p}=0,31\right.$; efeito moderado), com maior média para o jogo CT. Além disso, observaram-se valores significativos para o efeito principal resultado das finalizações $\left(p=0,001 ; \eta^{2} p=0,58\right.$; efeito moderado), com maior média para o resultado da ação: "fora".

Tabela 3: Médias (desvio-padrão) dos resultados das finalizações.

\begin{tabular}{lcccccc}
\hline Resultado & Gol & Trave & DG & RG & ID & Fora \\
\hline ST & $5,00(2,52)$ & $0,50(0,54)$ & $4,16(1,47)$ & $7,00(2,00)$ & $7,33(3,78)$ & $7,33(3,14)$ \\
\hline CT & $7,50(1,73)$ & $1,75(2,22)$ & $7,50(1,29)$ & $9,25(4,35)$ & $9,70(4,81)$ & $15,00(6,68)$
\end{tabular}

Efeito principal condição

$\mathrm{CT}>\mathrm{ST}$ 
Efeito principal resultado

Gol, DG, RG, ID e Fora > Trave; Fora > Go DG;

Legenda: ST = sem a permissão da "tiradinha"; CT= com a permissão da "tiradinha". DG= Defesa goleiro; RG = Rebote goleiro; ID = Interceptação defesa.

Fonte: Autores

De acordo com a tabela 4 , observou-se efeito de interação entre as variáveis $\left(p=0,02 ; \eta^{2} p=0,22\right.$; efeito pequeno), com maior média para os jogos CT, nas situações: laterais, escanteios e bola roubada.

Tabela 4: Média (desvio padrão) das condições de jogo entre cada situação de jogo.

\begin{tabular}{lcccccc}
\hline Situação & JO & CA & LAT & ESC & FAL & BR \\
\hline ST & $\begin{array}{c}9 \\
(4,6)\end{array}$ & $3(1,7)$ & $\begin{array}{c}3,16 \\
(1,47)\end{array}$ & $3(2,3)$ & $\begin{array}{c}2,6 \\
(2,6)\end{array}$ & $10,5(1,3)$ \\
\hline CT & $\begin{array}{r}11,5 \\
(3,1)\end{array}$ & $5,5(1,2)$ & $10,5(3)$ & $7(3,5)$ & $2(1,4)$ & $17,7(4,9)$ \\
\hline Efeito Interação p-valor & 0,17 & 0,17 & $0,001^{*}$ & $0,03^{*}$ & 0,71 & $0,001^{*}$ \\
\hline Tamanho do efeito $\left(\eta_{\mathrm{p}}{ }^{2}\right)$ & 0,03 & 0,03 & 0,25 & 0,09 & 0,003 & 0,24 \\
\hline Post-Hoc & - & - & $\mathrm{CT}>\mathrm{ST}$ & $\mathrm{CT}>\mathrm{ST}$ & & $\mathrm{CT}>\mathrm{ST}$ \\
\hline
\end{tabular}

Legenda: ST = sem a permissão da "tiradinha"; CT= com a permissão da "tiradinha"; JO = jogo organizado; CA = contraataque; $\mathrm{LAT}=$ lateral; $\mathrm{ESC}=$ escanteio; $\mathrm{FAL}=$ falta; $\mathrm{BR}=$ bola roubada.

Fonte: Autores

\section{Discussão}

O presente estudo comparou a interação das equipes, por meio da SNA, e a frequência de finalizações em diferentes setores da quadra, situações de jogo (contra-ataque, laterais, jogo organizado, falta) e resultados (gol, trave, defesa do goleiro, fora) em jogos de futsal da categoria sub-09, com e sem a permissão da regra da "tiradinha". Os resultados demonstraram maior interação das equipes nos jogos CT, refutando a hipótese estabelecida. Além disso, observou-se maior número de finalizações em situações originadas dos laterais e escanteios nos jogos CT, corroborando com a hipótese inicial. Ainda, independente da regra da "tiradinha", observou-se maior incidência de finalizações nos setores centrais e laterais da quadra e maior incidência de finalizações malsucedidas (fora e intercepção da defesa) do que bem-sucedidas. 
Observa-se que a manipulação de tarefas permite aumentar o nível de rendimento da equipe (MACHADO e colaboradores, 2016). Especificamente, hipotetizou-se que nos jogos ST jogadores aumentariam a incidência das ações de passes, elevando a interação da equipe. Entretanto, observou-se maior interação nos jogos CT. A dificuldade de a defesa impedir o gol, a partir da "tiradinha", conduz os adversários para áreas próximas da própria meta, aumentando o espaço na quadra para as trocas de passes do ataque. Além disso, outras estratégias são usadas para evitar o gol na "tiradinha" como, por exemplo, a defesa se posicionar entre o gol e o finalizador para bloquear o chute. Mesmo diante disso, possibilita-se que o ataque realize outras manobras ofensivas coletivas, como a tabela. Por outro lado, sugere-se que os jogos com a restrição da "tiradinha" conduziram a defesa a uma maior aproximação dos atacantes, o que reflete maior pressão, dificultando a troca de passes do ataque. Essa dificuldade se acentua diante do estágio de desenvolvimento motor (inicial) (GALLAHUE; OZMUN, 2001) em que se encontram os jogadores da categoria sub-09.

Os setores centrais da quadra se apresentam como locais relevantes para os gols no futsal (CUZOVIC; SOLAK, 2018). Os resultados do presente estudo apontaram para uma maior incidência de finalizações nos setores centrais e laterais da quadra, independente da condição do jogo (CT e ST). Sugere-se que as possíveis dificuldades motoras (COSTA e colaboradores, 2010; MÜLLER; DA COSTA; GARGANTA, 2018) e as ações coletivas (GARGANTA, 2002), para superar o adversário na categoria sub-09, aliadas à possibilidade de obtenção de gols facilmente, em razão da baixa estatura dos goleiros, conduziram os jogadores a priorizarem as ações de finalização, até mesmo em setores com maior dificuldade de sucesso (laterais) (CABRAL e colaboradores, 2013; PRAÇA; CABRAL; GRECO, 2013), independente da possibilidade de usar a "tiradinha".

Além disso, observou-se maior incidência de finalizações provenientes das ações de laterais e escanteios com baixa eficácia nos resultados dos chutes, independente da permissão da "tiradinha". Conforme sugerido no parágrafo anterior, as ações de finalização se destacaram nos jogos da categoria sub-09. Reconhecendo isso, sugere-se que a distância exigida pela regra (5 metros) nas cobranças de laterais e escanteios represente uma condição propícia para a finalização, tanto para os jogos CT (possibilidade da ação de recepção antes da finalização), quanto para os jogos ST. A realização de uma ação motora proveniente de uma situação de bola parada (escanteios ou laterais), no futsal, representa uma estratégia oportuna para atingir a meta adversária facilmente (CAMPOS, 2014; FUKUDA; SANTANA, 2012), devido ao foco atencional dos jogadores se direcionar apenas para a bola e para realização da ação motora (WILLIAMS; HODGES, 2005). Entretanto, espera-se que, nas categorias iniciais, o resultado dessa ação apresente baixa efetividade diante das variações (instabilidades) na 
execução dos movimentos, provenientes da fase de desenvolvimento da idade (GALLAHUE; OZMUN, 2001).

O presente estudo apresenta limitações. Não foi possível padronizar as dimensões das quadras para os jogos selecionados, o que pode ter impactado, por exemplo, a incidência nas ações de finalizações e passes entre os jogadores. Além disso, a amostra do presente estudo apresentou diferentes níveis competitivos. Neste sentido, deve-se ter cautela na transferência dos resultados para equipes da mesma idade (sub-09). Sugere-se que futuros estudos manipulem esses aspectos a fim de reduzir as possíveis influências.

\section{Conclusão}

Conclui-se que a presença da regra da "tiradinha", nos jogos de futsal, na categoria sub-09, aumenta a interação entre os jogadores. Além disso, os jogadores utilizaram essa regra para o aumento das ações de finalização. A partir disso, sugere-se que treinadores possam utilizar a regra da "tiradinha", no processo de treino, para aumentar a participação dos jogadores, por meio das trocas de passes, bem como para direcionar os jogadores aos sinais relevantes na execução técnica do chute e em outros elementos (goleiro, colegas de equipes e adversários). Porém, pondera-se que treinadores possam também refletir acerca dos efeitos, a longo prazo, desta regra na formação de jovens jogadores. Por exemplo, poderá criar uma rigidez técnico-tática, prejudicando a construção de um acervo rico e diversificado essencial para as resoluções dos problemas emergentes no jogo. Além disso, a centralização em alguns jogadores (maior nível maturacional) para as cobranças da "tiradinha" poderá reduzir as oportunidades de ação para os demais jogadores da equipe, impactando no desenvolvimento de outras ações técnico-táticas fundamentais para a idade. As alterações de regras nas competições (por exemplo, a ausência da "tiradinha" e a obrigatoriedade da permanência do arremesso de meta na quadra defensiva), nas tarefas, nos treinamentos e nas categoriais iniciais, fazem-se necessárias para a criação de um ambiente propício e estimulante para o desenvolvimento dos jogadores.

\section{Referências}

AGRAS, H.; FERRAGUT, C.; ABRALDES, J. A. Match analysis in futsal: A systematic review. International Journal of Performance Analysis in Sport. v. 16, n. 2, p. 652-686, 2016. 
CABRAL, F. A.; PRAÇA, G. M.; TEIXEIRA, C. S.; MATIAS, C. J. A. S; GRECO, P. J. Análise de jogo no futsal escolar: situação e efetividade de finalização. Revista Mineira de Educação Física. v. Edição Especial, n. 9, p. 408-414, 2013.

CAMPOS, J. Análise dos Gols em Jogos de Futsal Sub-17 no Campeonato Estadual de São Paulo 2012. Revista Brasileira de Futsal e Futebol. v. 6, n.19, p. 27-31, 2014.

CASO, S.; VAN DER KAMP, J. Variability and creativity in small-sided conditioned games among elite soccer players. Psychology of Sport and Exercise. v. 48, p. 101645, 2020.

CASTAGnA, C; D'OTTAVIO, S.; VERA, J. G.; ÁLVARES, J. C. B. Match demands of professional Futsal: A case study. Journal of Science and Medicine in Sport. v. 12, n. 4, p. 490-494, 2009.

CBFS. Livro Nacional de Regras 2015. Fortaleza, 2015. Disponível em: https://www.cbfs.com.br/futsalregras Acesso em 13 agosto 2021.

CLEMENTE, Filipe Manuel; MARTINS, Fernando Manuel Lourenço; MENDES, Rui Sousa. Social Network Analysis applied to team sports analysis. London: Springer, 2016.

COHEN, J. Statistical power analysis for the behavioral sciences. 2. ed. New York: Lawrence Erlbaum, 1988.

COSTA, I. T.; GARGANTA, J.; GRECO, P. J.; MESQUITA, I.; AFONSO, J. Assessment of tactical principles in youth soccer players of different age groups. Revista Portuguesa de Ciências do Desporto. v. 10, n. 1, p. 147-157, 2010.

CUZOVIC, D.; SOLAK, Z. Characteristics of goals and game at the top championships in futsal. Revista Brasileira de Futsal e Futebol. v. 10, n. 38, p. 271-277, 2018.

DOGRAMACI, S. N.; WATSFORD, M. L.; MURPHY, A. J. Time-motion analysis of international and national level futsal. Journal of Strength and Conditioning Research. v. 25, n. 3, p. 646-651, 2011.

FARIAS, A. B. Competições esportivas como meio de educação e formação de crianças e jovens: um estudo sobre as categorias sub 9 e sub 11 de futsal. 2016. 51 f. Trabalho de Conclusão de Curso, Universidade Federal do Rio Grande do Sul, Rio Grande do Sul, 2016.

FERGUSON, C. J. An effect size primer: A guide for clinicians and researchers. Professional Psychology: Research and Practice. v. 40, n. 5, p. 532-538, 2009.

FUKUDA, S. J. P.; SANTANA, W. C. Análises dos gols em jogos da liga futsal 2011. Revista Brasileira de Futsal e Futebol. v. 4, n. 11, p. 62-66, 2012.

GALlAHUE, David L.; OZMUN, John C. Compreendendo o desenvolvimento motor: bebês, crianças, adolescentes e adultos. 1. ed. São Paulo: Phorte Editora, 2001.

GARGANTA, J. Competências no ensino e treino de jovens futebolistas. Lecturas Educación Física y Deportes. v. 8, n. 45, p. 1-15, 2002. 
LANDIS, J. R.; KOCH, G. G. The measurement of observer agreement for categorical data. Biometrics. v. 33, n. 1, p. 159, 1977.

LAPRESA, D.; ÁLVAREZ, L.; ARANA, J.; GARZÓN, B.; CABALLERO, V. Observational analysis of the offensive sequences that ended in a shot by the winning team of the 2010 UEFA Futsal Championship. Journal of Sports Sciences. v. 31, n. 15, p. 1731-1739, 2013.

MACHADO, J. C.; ALCÂNTARA, C.; PALHETA, C.; SANTOS, J. O. L.; BARREIRA, D.; SCAGLIA, A. The influence of rules manipulation on offensive patterns during small-sided and conditioned games in football. Motriz. v. 22, n. 4, p. 290-298, 2016.

MALINA, R. M.; PEÑAREYES, M. E.; EISENMANN, J. C.; HORTA, L.; RODRIGUES, J.; MILLER, R. Height, mass and skeletal maturity of elite portuguese soccer players aged 11-16 years. Journal of Sports Sciences. v. 18, n. 9, p. 685-693, 2000.

MÜLlER, E. S.; COSTA, I. T.; GARGANTA, J. Tactical analysis in futsal: comparative study of performance by players from four age levels. Revista Brasileira de Ciencias do Esporte. v. 40, n. 3, p. 248-256, 2018.

PRAÇA, G. M.; CABRAL, F. DE A.; GRECO, P. J. Comparação do padrão de finalização de praticantes de futsal de diferentes idades. Conexões. v. 11, n. 4, p. 24-44, 2013.

SILVA, M.; RÉ, A. H. N.; MATIAS, C. J. A. S.; GRECO, P. J. Estratégia e Tática no futsal: uma análise crítica. Caderno de Educação Física e Esporte. v. 10, n. 19, p. 75-84, 2011.

TABACHNICK, Barbara G.; FIDELL, Linda S.; ULLMAN, Jodie B. Using Multivariate Statistics. Boston: Pearson, 2007.

TRAVASSOS, B. et al. Adaptive behaviours of attacking futsal teams to opposition defensive formations. Human Movement Science. v. 47, p. 98-105, 2016.

WILLIAMS, A. M.; HODGES, N. J. Practice, instruction and skill acquisition in soccer: Challenging tradition. Journal of Sports Sciences. v. 23, n. 6, p. 637-650, 2005.

\section{Como citar este artigo}

MACEDO, R. G.; MOREIRA, P. D.; PRAÇA, G. M. A proibição de chute após a cobrança do lateral ou escanteio modifica o comportamento de jogadores de futsal da categoria sub-09? Revista Kinesis, Santa Maria, v. 39, p.1-13, 2021.

*O presente trabalho não contou com apoio financeiro de nenhuma natureza para sua realização. 